\title{
Study on Anomalous Scaling Exponents for Molecular Thin Film Growth Using Surface Lateral Diffusion Model
}

\author{
Hyejin Gong and Sanggyu Yim* \\ Department of Chemistry, Kookmin University, Seoul 136-702, Korea. *E-mail: sgyim@kookmin.ac.kr \\ Received April 7, 2011, Accepted May 18, 2011
}

\begin{abstract}
Anomalous scaling behaviors such as significantly large growth exponent $(\beta)$ and small reciprocal of dynamic exponent $(1 / z)$ values for many molecular crystalline thin films have been reported. In this study, the variation of scaling exponent values and consequent growth behaviors of molecular thin films were more quantitatively analysed using a (1+1)-dimensional surface lateral diffusion model. From these simulations, influence of step edge barriers and grain boundaries of molecular thin films on the various scaling exponent values were elucidated. The simulation results for the scaling exponents were also well consistent with the experimental data for previously reported molecular thin film systems.
\end{abstract}

Key Words : Molecular thin film, Growth behavior, Scaling exponent, Lateral diffusion model

\section{Introduction}

Control over molecular thin film growth is of considerable interest for a wide range of (opto)electronic device applications such as small molecule organic photovoltaics $(\mathrm{OPVs}) .^{1-3}$ Especially, thin film growth behavior and consequent surface morphology of molecular crystals play a crucial role in determining the performance of devices, such as charge carrier mobility and current-voltage characteristics. ${ }^{4}$ In contrast to well-studied atomic-based inorganic thin films, however, current knowledge on the growth mechanism of molecular thin films is limited. Recently several studies have attempted to understand the growth behavior of molecular thin films, using a scaling theory, ${ }^{5-8}$ which is a commonly used method for studying the growth dynamics of inorganic thin film systems. ${ }^{9-12}$ These studies were carried out using a combination of experimental scanning probe methods such as atomic force microscopy (AFM) and theoretical scaling calculations such as height difference correlation function (HDCF) analysis, which provides a variety of scaling exponents, such as roughness $(\alpha)$, growth $(\beta)$ and dynamic $(z)$ exponent, and the relationship between them. Two unique characteristics of molecular thin film growth compared to inorganic thin film systems have been reported. One is the large growth exponent $(\beta)$ values, indicating rapid surface roughening during the deposition. The other is the pronounced small reciprocal of dynamic exponent $(1 / z)$ values. Consequently these two anomalous exponent values resulted in a significant deviation from the conventional scaling law, $\beta / \alpha=1 / z$. It has been suggested that the rapid roughening and large $\beta$ values can be explained by spatial inhomogeneities between the crystalline domains in molecular thin films, which results from the structural anisotropy of the molecular materials. ${ }^{5,14}$ For the anomalous scaling behavior of molecular thin films, it has been suggested that high potential energy barriers at the step edges of crystalline mounds in molecular thin films led to the upward growth and consequent small $1 / z$ values. ${ }^{6}$ In spite of the experimental data and plausible suggestions, however, the factors that influence molecular thin film growth were not fully understood yet. This is partly because the analyses were performed for individual thin film growth and hence there were few studies on the system in which all the parameters affecting the growth behaviors were varied.

In this study, we conducted the simulations for a molecular thin film growth using a simple (1+1)-dimensional surface lateral diffusion model, varying simultaneously the two parameters related to step-edge barriers and grain boundaries. The results could provide more comprehensive and systematic insight into the role of the parameters on the scaling exponents and growth behaviour of the molecular crystalline films.

\section{Theory and Modeling}

The growth behavior and surface morphology of thin films can be analyzed through HDCF analysis, ${ }^{9-13}$ where the $\alpha, \beta$ and $z$ exponents can be determined from the mean square surface fluctuation, $g(R)$;

$$
\begin{gathered}
g(R)=\left\langle\left[h(x)-h\left(x^{\prime}\right)\right]^{2}\right\rangle \\
R=x-x^{\prime}
\end{gathered}
$$

The average is taken over all pairs of surface points separated laterally by the length, $R$. There are two distinct regimes which depend on the relative magnitudes of $R$ and the correlation length, $\xi ; g(R) \propto R^{2 \alpha}$ for $R<\xi \xi$ and $g(R)$ $=2 \sigma^{2}$ for $R \gg \xi$, where $\sigma$ is the mean-square surface roughness. The correlation length, $\xi$, at each thickness is determined by fitting the HDCF to the following analytical function: $g(R)=2 \sigma^{2} \tilde{g}(R / \xi)$ where $\tilde{g}(x)=1-\exp \left(-x^{2 \alpha}\right) .{ }^{15}$ The parameters $\sigma$ and $\xi$ are related to the film thickness, $D$, according to the power laws, $\sigma \propto D^{\beta}$ and $\xi \propto D^{1 / z}$. It has been shown that in many cases, in particular for the growth 

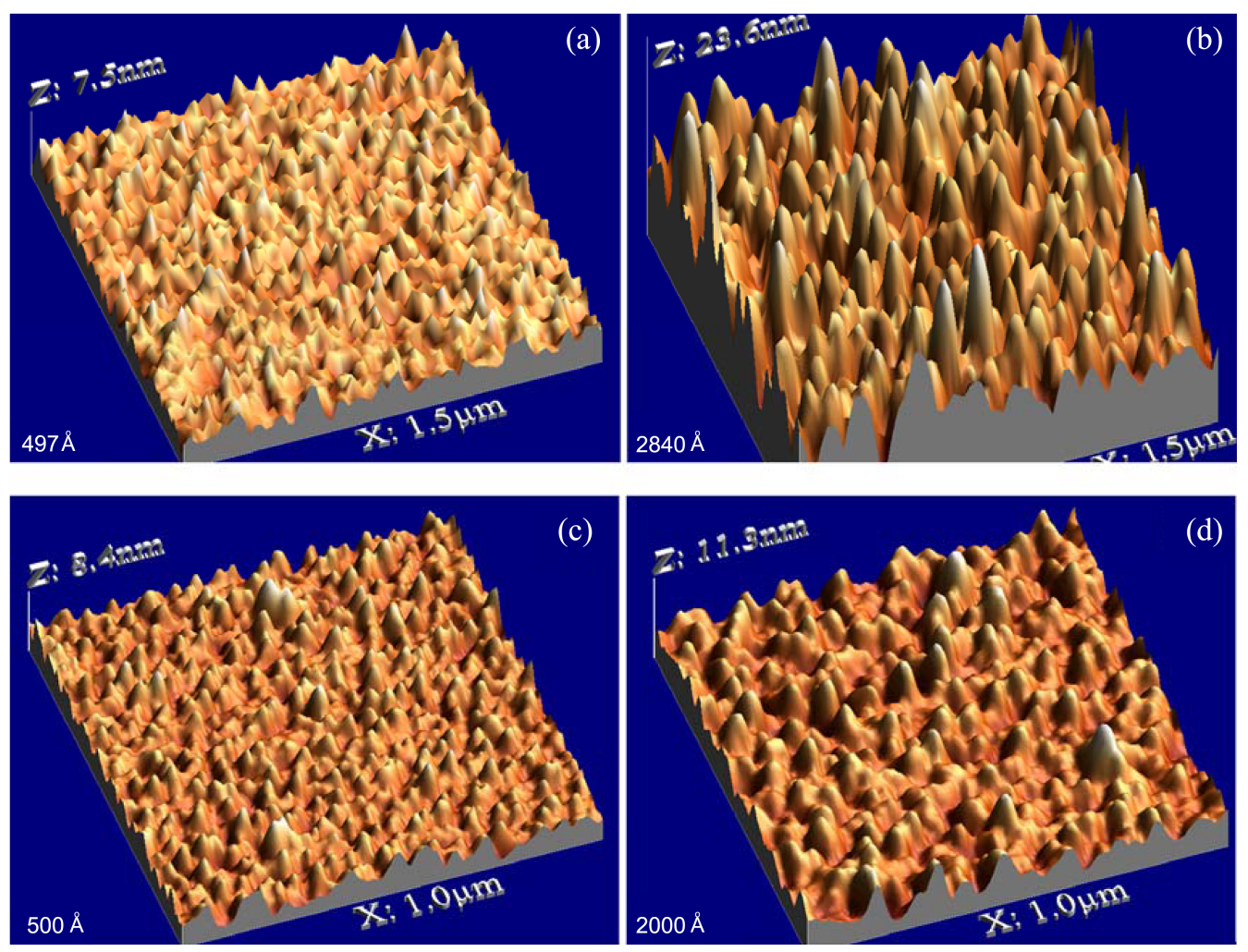

Figure 1. AFM images of PTCDA thin films with thickness (D) of (a) $497 \AA$ and (b) $2840 \AA$; and for $\mathrm{C}_{60}$ thin films with $D$ of (c) $500 \AA$ and (b) $2000 \AA$.

of most inorganic thin films, the $\alpha, \beta$ and $z$ values are not independent and obey the conventional scaling law, $1 / z \cong \beta / \alpha \cdot{ }^{14,16,17}$ In order to evaluate the degree of deviation from the conventional scaling laws, the anomaly value, $\rho$, was defined as follows, $\rho=(1 / z) /(\beta / \alpha)$. For an ideal system that perfectly obeys the conventional scaling laws, $\rho$ approaches one. In the case of molecular thin films, however, a broad range of significantly smaller $\rho$ values have been reported such as the case for 3,4,9,10-perylenetetracarboxylic dianhydride (PTCDA) $(\rho=0.33),{ }^{6}$ zinc phthalocyanine $(\mathrm{ZnPc})(\rho=0.54)^{8}$ and $\mathrm{C}_{60}(\rho=0.67){ }^{7}$ The surface morphology evolutions for the PTCDA and $\mathrm{C}_{60}$ thin films shown in Figure 1 seemed to be related to the $\rho$ values, i.e. the smaller the $\rho$ value, the spikier the film grows. In order to more quantitatively analyze this behavior, simulations of $(1+1)$ dimensional film growth were conducted using a surface lateral diffusion model in this work.

Organic molecules are bonded to each other by relatively weak van der Waals forces which cause the impinging molecules to diffuse laterally across the surface. In our simulations, impinging particles were allowed to diffuse along the surface up to a finite distance (ten columns maximum) before stopping when they were in an energetically stable position such as A or B as shown in Figure 2. The positions $\mathrm{A}$ and $\mathrm{B}$ are equivalent, but in order for the impinging particle to diffuse down to the position $\mathrm{B}$, it must overcome and additional energy barrier which exists at the step-edge; so-called Schwoebel barrier or step-edge barrier. Observation of surface morphology evolution and determination of

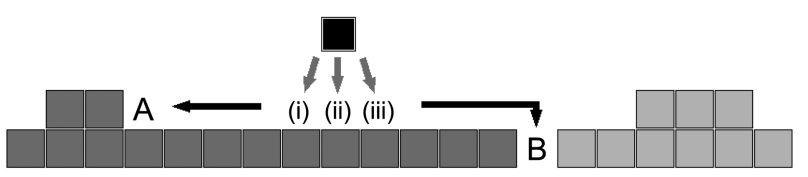

Figure 2. Schematic of the simple (1+1)-dimensional film growth model. A deposited particle (black square) diffuses along the surface before stopping when they are in an energetically stable position. (see text)

scaling exponents were carried out varying two parameters, $P F$ and $p_{b t w}$. The preference factor, $P F=n$, was defined when the deposited particle was in a position where diffusion to a step edge in the same layer was $n$ columns greater than diffusion down to a step edge in the lower layer, with equal probability of diffusion occurring in the two directions. In the case of $P F=0$, for example, the impinging particle at position (ii) in Figure 2 has equal probability of moving left and right since both involve travelling 5 columns to find a stable position (A and B). A particle deposited at (i) and (iii) moves left and right, respectively. However, when $P F=2$, an impinging particle deposited at (iii) has an equal probability of moving in both directions since from this position diffusion in the same layer ( 6 columns to A) is 2 columns greater than diffusion down to the lower layer (4 columns to B). In this case, a particle deposited at (i) or (ii) moves left. Consequently, an increase in the $P F$ values in the simulations leads to the preferential sticking of the deposited particle towards the stable position in the same layer, which produces the same effect as an increase in potential energy 
barriers at the step edges. ${ }^{6}$ The other parameter, $p_{b t w}$ was defined as the probability that the position between the grains with different orientations, e.g., position $\mathrm{B}$ in Figure 2 , accommodated the particle. For example, when $p_{b t w}=1$, the particle can occupy the position without any restrictions. In contrast, when $p_{b t w}=0$, the particle is not allowed to fall into the position at all. Therefore, in the simulations, a decrease in $p_{b t w}$ causes the evolution of deep grain boundaries between the crystalline domains with different orientations.

\section{Results and Discussion}

Figure 3 shows representative evolution of surface morphologies with various $p_{b t w}$ and PF values. For each simulation,
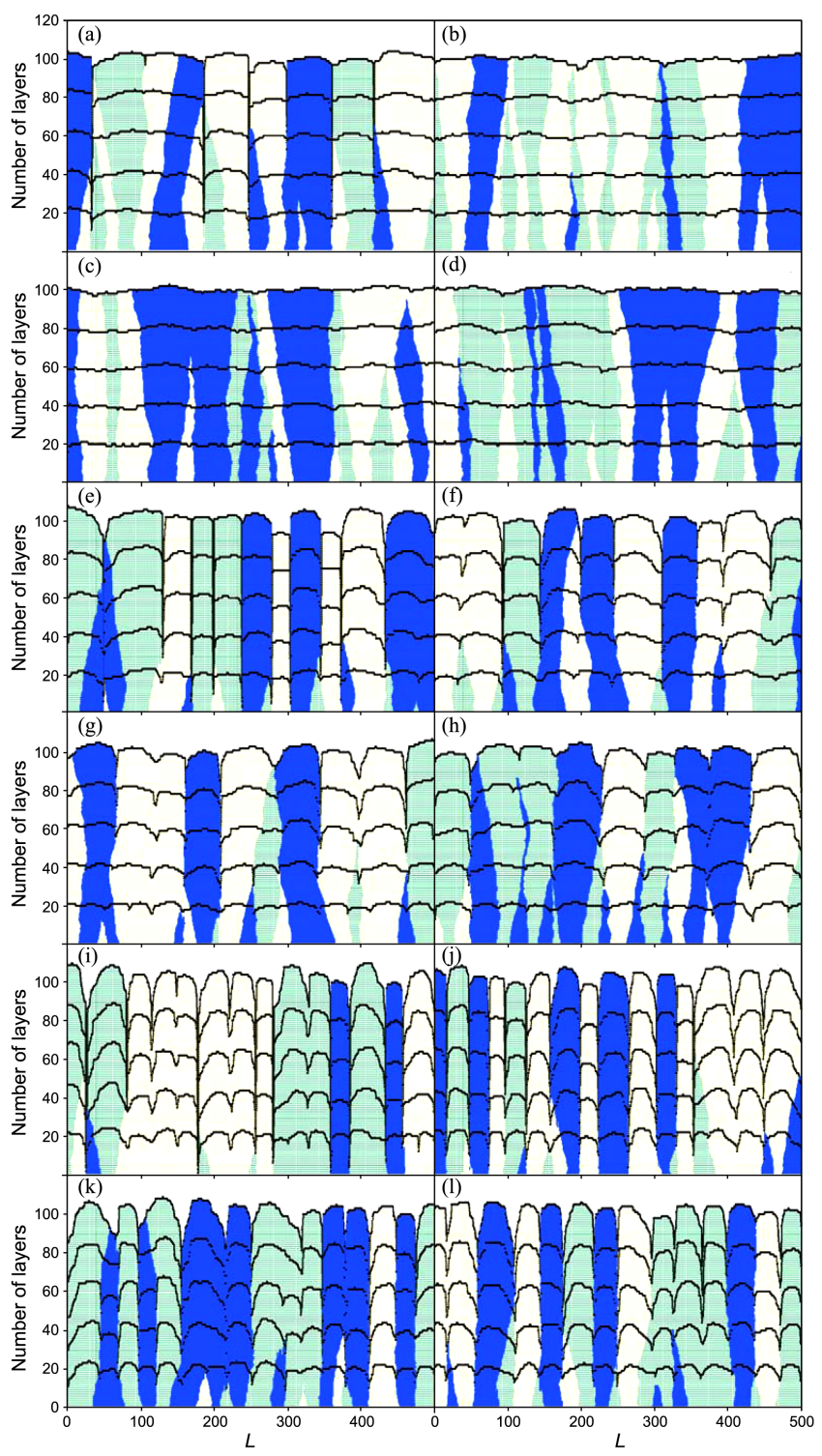

Figure 3. Representative surface growth patterns generated by simulations at (a) $p_{b t w}=0.05$ and $P F=0$, (b) $p_{b t w}=0.35$ and $P F=$ 0 , (c) $p_{b t w}=0.65$ and $P F=0$, (d) $p_{b t w}=0.95$ and $P F=0$, (e) $p_{b t w}=$ 0.05 and $P F=2$, (f) $p_{b t w}=0.35$ and $P F=2$, (g) $p_{b t w}=0.65$ and $P F$ $=2$, (h) $p_{b t w}=0.95$ and $P F=2$, (i) $p_{b t w}=0.05$ and $P F=4$, (j) $p_{b t w}=$ 0.35 and $P F=4,(\mathrm{k}) p_{b t w}=0.65$ and $P F=4$, and (1) $p_{b t w}=0.95$ and $P F=4$.
50,000 particles were randomly deposited on a substrate consisting of 500 horizontal columns, $L=500$. In Figures $3(\mathrm{a})-3(\mathrm{~d})$, the $p_{b t w}$ values were $0.05,0.35,0.65$ and 0.95 , respectively and the $\mathrm{PF}$ value was fixed to 0 , i.e. no step edge barrier existed. In all the figures, thick curves were drawn after the deposition of each set of 10,000 particles so that the increase in roughness and the evolution of grain boundaries could be easily recognized. It was also assumed that the particles were allowed to have three different molecular orientations which were differentiated by different shadings in the figures. The initially deposited particles could randomly adopt one of the three orientations and subsequent particles that stacked on existing grains by lateral diffusion had the same orientation as the initial grains. In the case of $p_{b t w}=0.05$, implying that the impinging molecules could hardly occupy the position between two adjacent grains with different orientation, small number of deep grain boundaries were formed as shown in Figure 3(a). However, when $p_{b t w} \geq 0.3$, deep grain boundaries were barely observed (Figs. 3(b)-3(d)). The $\beta, 1 / z$ and $\rho$ values when $P F=0$ were plotted as a function of $p_{b t w}$ in Figures 4(a)-4(c), respectively. Figure 4(a) shows that the growth exponents $(\beta)$ were

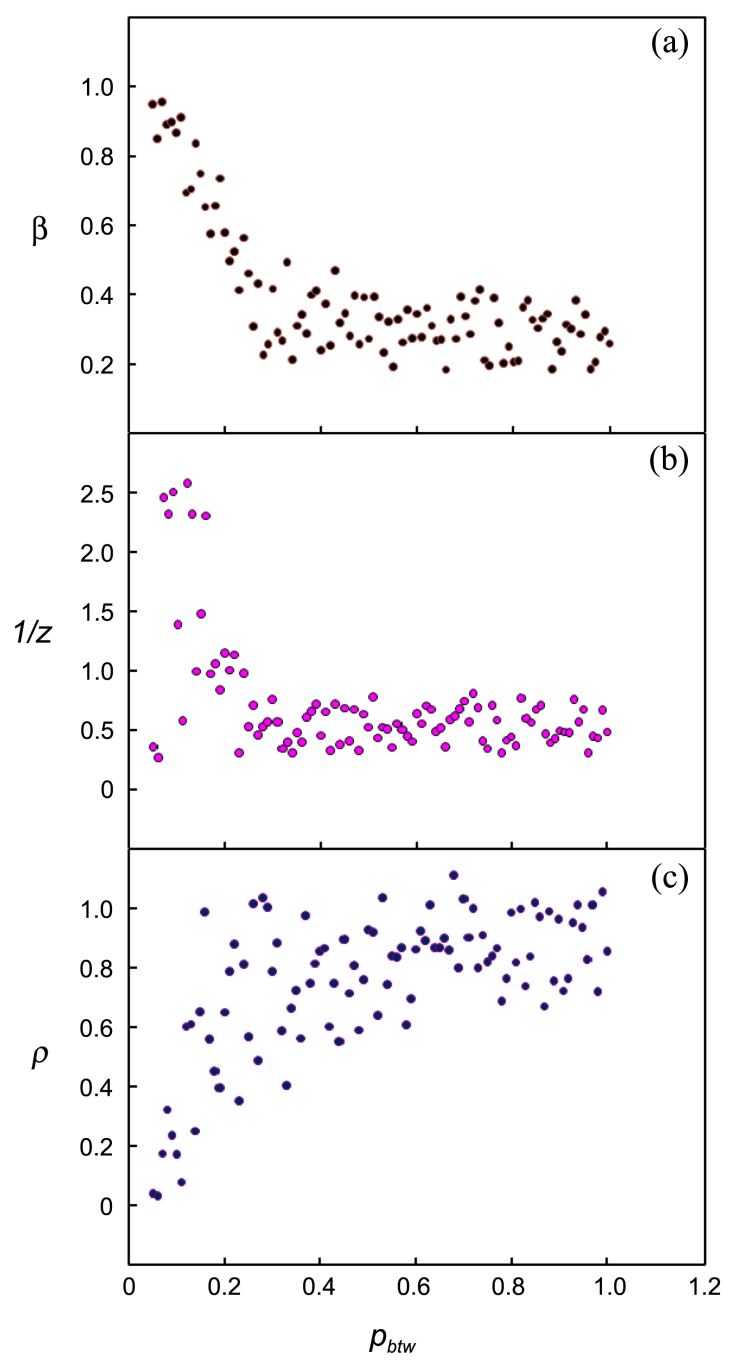

Figure 4. Plots of (a) $\beta$, (b) $1 / z$ and (c) $\rho$ value as a function of $p_{b t w}$ when $P F=0$. 
strongly dependent on the $p_{b t w}$ values. When $p_{b t w} \geq 0.3$, the $\beta$ values were stabilized to $0.2-0.4$, consistent with those observed in many conventional inorganic thin film systems. However, when $p_{b t w}$ value is smaller than 0.3 , the $\beta$ values increased very rapidly. This indicates that the significantly large $\beta$ values observed for molecular thin film systems can be explained by the evolution of deep grain boundaries. The $1 / z$ values were around 0.5 when $p_{b t w} \geq 0.3$, as shown in Figure 4(b). The relatively large variation in $1 / z$ values at very small $p_{b t w}$ values might be caused by the difficulty in estimating the correlation lengths $(x)$ due to the deep grain boundaries. Figure 4(c) shows that the anomaly $(\rho)$ values ranged from 0.6 to 1.1 when $p_{b t w} \geq 0.3$, indicating that the growth did not deviate significantly from the conventional scaling laws. In contrast, the evolution of surface morphology was significantly different when PF value increased. In Figures $3(\mathrm{e})-3(\mathrm{~h})$, the $p_{b t w}$ values were $0.05,0.35,0.65$ and 0.95 , respectively and the PF value was fixed to 2 . In all cases, the evolution of deep grain boundaries was clearly observed regardless of $p_{b t w}$ values. The increase in the mound number density and a pronounced upward growth were also observed. Continuous increase in mound number density and upward growth was found as PF value increased, as shown in Figures 3(i)-3(1), where the $p_{b t w}$ values were 0.05, $0.35,0.65$ and 0.95 , respectively and the PF value was 4 . In order to analyse the effect of the two parameters on the evolution of surface roughness and anomalous growth behaviour, the growth exponent $(\beta)$ and anomaly $(\rho)$ values for these non-zero $P F$ models were plotted as a function of $p_{b t w}$ in Figure 5 and Figure 6, respectively. Figure 5 shows that as $P F$ increases, the growth exponent $(\beta)$ values were stabilized at gradually higher levels. The average $\beta$ values were $0.56,0.69$ and 0.74 for $P F=1$ (Fig. 5(a)), 2 (Fig. 5(b)), and 3 (Fig. 5(c)), respectively. These values represent a gradual increase in $\beta$ values from the average value of 0.40 for $P F=0$ as shown in Figure 4(a). In the case of anomaly $(\rho)$ values, a more dramatic change was observed as $P F$ increases (Fig. 6). The average $\rho$ values decreased very rapidly to $0.30,0.07$ and 0.02 for $P F=1$ (Fig. 6(a)), 2 (Fig. 6(b)), and 3 (Fig. 6(c)), respectively, compared to the

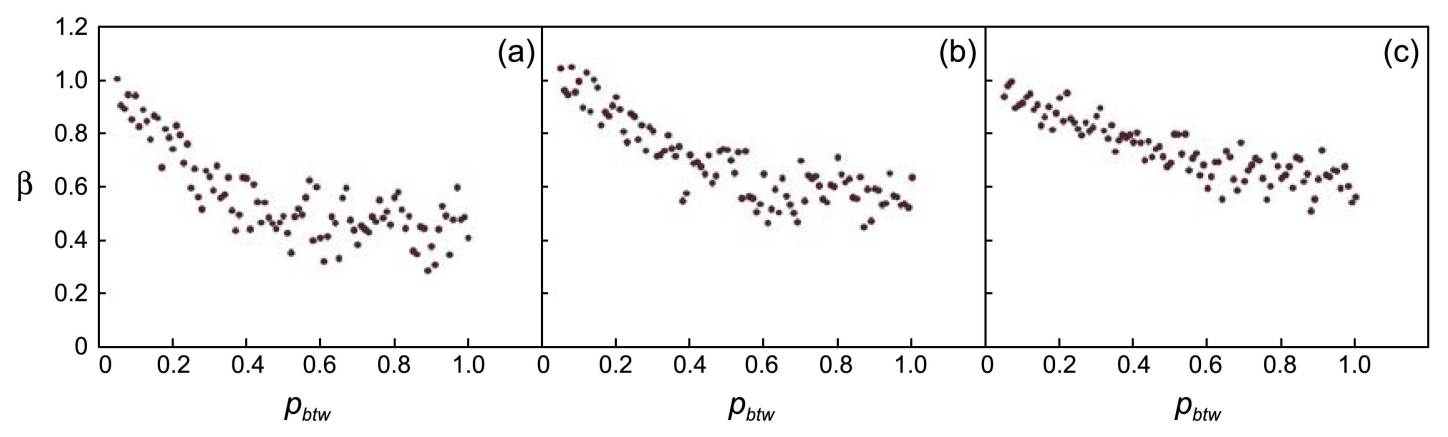

Figure 5. Plots of $\beta$ values as a function of $p_{b t w}$. The $P F$ values were (a) 1 , (b) 2 and (c) 3 .

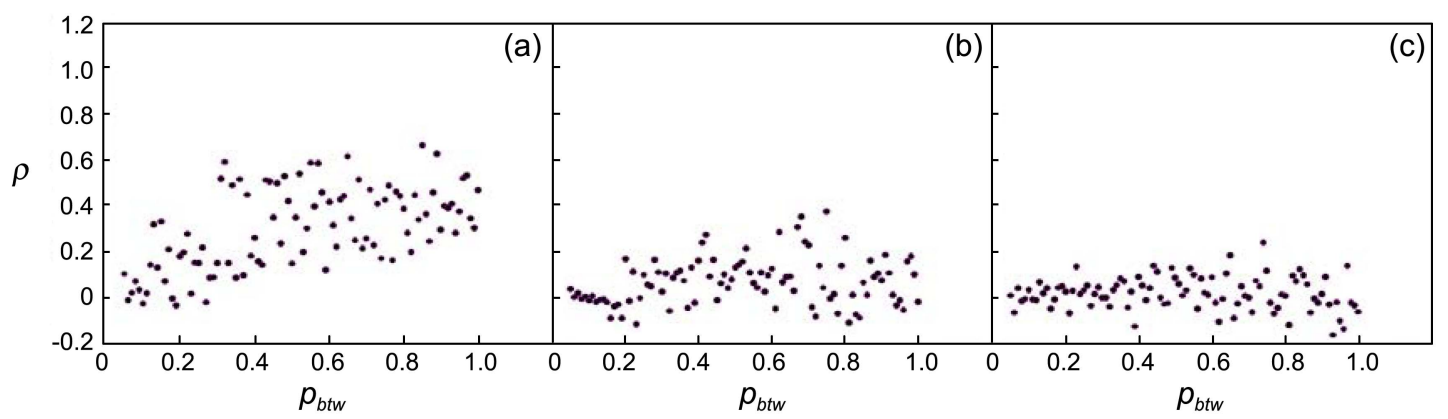

Figure 6. Plots of $\rho$ values as a function of $p_{b t w}$. The $P F$ values were (a) 1, (b) 2 and (c) 3 .

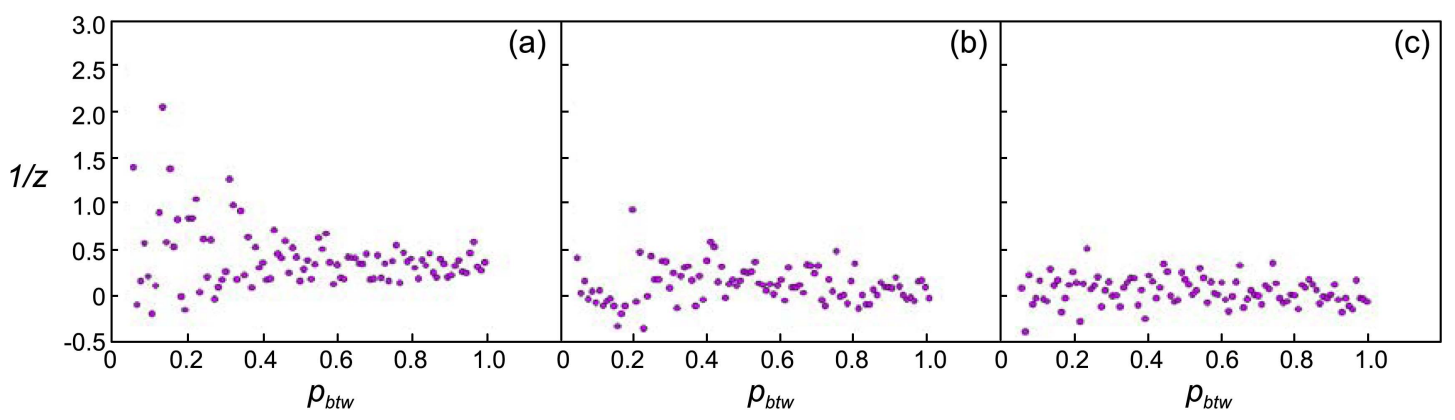

Figure 7. Plots of $1 / z$ values as a function of $p_{b t w}$. The $P F$ values were (a) 1, (b) 2 and (c) 3 . 
average $\rho$ value of 0.75 for $P F=0$ as shown in Figure 4(c). However, the rapid decrease in $\rho$, i.e. large deviation from the conventional scaling laws, is likely attributed to not only the increase in $\beta$ values but also the decrease in $1 / z$ values as $P F$ increases (Fig. 7). The $1 / z$ values were shown to approach 0 rapidly as $P F$ increased. The average $1 / z$ values were $0.42,0.12$ and 0.05 for $P F=1$ (Fig. 7(a)), 2 (Fig. 7(b)), and 3 (Fig. 7(c)), respectively, compared to the average $1 / z$ value of 0.71 for $P F=0$ as shown in Figure 4(b). The simulations, therefore, indicated that the anomalous scaling behavior occurred when the potential energy barriers at the step edges of pre-existing molecular islands were large although the impinging particle could occupy the position between grain boundaries without any restriction. The average $\alpha, \beta, 1 / z$ and $r$ values obtained from the simulations were plotted as a function of $P F$ in Figure 8(a). The most interesting aspect of this analysis is the fact that $1 / z$ value decreases rapidly as $P F$ increases before finally converging to zero for the cases when $P F \geq 3$. These small $1 / z$ values result in pronounced anomalous scaling behaviors, i.e. significantly small $\rho$ values. The variation trend of simulated
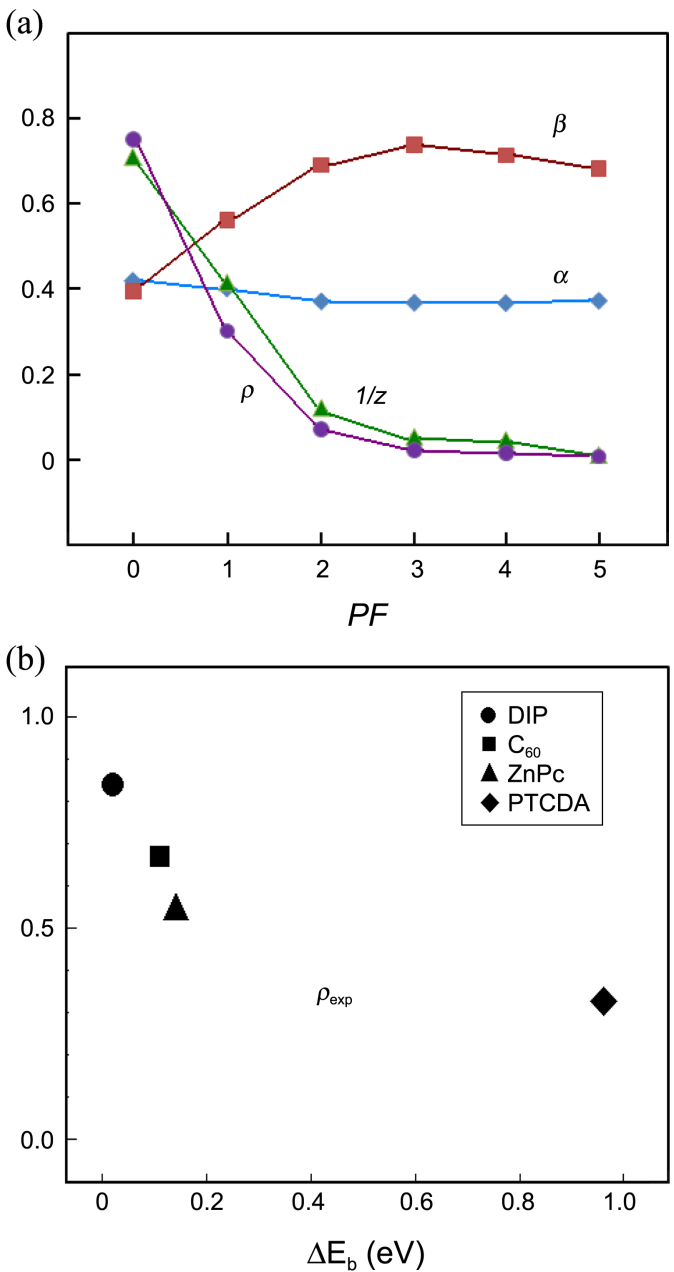

Figure 8. (a) Plots of average scaling exponents, $\alpha(\diamond), \beta(\boldsymbol{\square}), 1 / z$ $(\boldsymbol{\Delta})$, and $\rho(\mathbf{O})$ simulated as a function of $P F$ and (b) plots of the $\rho$ values obtained from experimental results as a function of step edge barriers $\left(\Delta E_{b}\right)$. anomaly values in Figure 8(a) is well consistent with that of the experimental results plotted as a function of step-edge barriers as shown in Figure 8(b). The evolution of surface morphologies of each molecular thin film system was well described in the literatures. ${ }^{6-8,14}$ The step-edge barriers $\left(\Delta E_{b}\right)$ for the molecular thin films were determined by intermolecular interaction energy calculations using molecular mechanics (MM) force field parameters, which were well described elsewhere. ${ }^{6} \Delta E_{b}$ values of $0.95,0.14,0.11$ and $0.02 \mathrm{eV}$ were determined for PTCDA, $\mathrm{ZnPc}, \mathrm{C}_{60}$ and diindenoperylene (DIP) respectively, a higher value indicating a greater decrease of the intermolecular interactions at the step edges. The anomaly $(\rho)$ values were determined from the exponent values taken from the literatures. ${ }^{6,8}$

In conclusion, the effects of step-edge barriers and grain boundaries on the anomalous scaling exponent values and growth behaviour of molecular thin films were estimated using the $(1+1)$-dimensional lateral diffusion model. In the simulations, the two parameters, $P F$ and $p_{b t w}$, that were related to step edge barriers and the evolution of deep grain boundaries, respectively, were varied simultaneously. The simulation results indicated that both of these parameters contributed to the large growth exponent, $\beta$, values. This can be explained by the fact that large step edge barriers promoted upward dominant growth and rapid roughening as $P F$ increases. The $\beta$ values also increased rapidly when the evolution of deep grain boundaries increased due to the small $p_{b t w}$ values. The anomaly, $\rho$, values, however, seemed to be more strongly dependent on the parameter $P F$ than $p_{b t w}$. For example, when $P F=0$, the simulation results showed conventional scaling behaviors, i.e. $\rho \approx 1$, except for the case that $p_{b t w}$ was significantly small. In contrast, even a slight increase in $P F$ led to a rapid decrease in the $r$ values through the whole range of $p_{b t w}$. This could be explained by the upward dominant growth and consequently small $1 / z$ values when the potential barriers at the step edge were large. In addition, the simulation results were well consistent with the experimental data for several molecular thin films systems with various step-edge barriers.

Acknowledgments. This work was supported by Basic Science Research Program through the National Research Foundation of Korea (NRF) funded by the Ministry of Education, Science and Technology (2010-0013057).

\section{References}

1. Gundlach, D. J.; Lin, Y. Y.; Jackson, T. N.; Nelson, S. F.; Schlom, D. G. IEEE Electron Device Lett. 1997, 18, 87.

2. Lunt, R. R.; Benziger, J. B.; Forrest, S. R. Adv. Mater. 2007, 19, 4229.

3. Rim, S. B.; Fink, R. F.; Schöneboom, J. C.; Erk, P.; Peumans, P. Appl. Phys. Lett. 2007, 91, 173504.

4. Sullivan, P.; Jones, T. S.; Ferguson, A. J.; Heutz, S. Appl. Phys. Lett. 2007, 91, 233114.

5. Yim, S.; Jones, T. S. Phys. Rev. B 2006, 73, 161305.

6. Yim, S.; Kim, K.-I.; Jones, T. S. J. Phys. Chem. C 2007, 111, 10993.

7. Yim, S.; Jones, T. S. Appl .Phys. Lett. 2009, 94, 021911. 
8. Kim, J.; Lim, N.; Park, C. R.; Yim, S. Surf. Sci. 2010, 604, 1143.

9. Krug, J. Adv. Phys. 1997, 46, 130.

10. Barabasi, A. L; Stanley, H. E. Fractal Concepts in Surface Growth; Cambridge University Press: 1995.

11. Biscarini, F.; Samorí, P.; Greco, O.; Zamboni, R. Phys. Rev. Lett. 1997, 78, 2389.

12. Zhao, Y.-P.; Fortin, J. B.; Bonvallet, G.; Wang, G.-C.; Lu, T.-M. Phys. Rev. Lett. 2000, 85, 3229.

13. Hong, D.; Do, Y. R.; Kwak, H. T.; Yim, S. J. Appl. Phys. 2011,
109, 063507.

14. Dürr, A. C.; Schreiber, F.; Ritley, K. A.; Kruppa, V.; Krug, J.; Dosch, H.; Struth, B. Phys. Rev. Lett. 2003, 90, 016104.

15. Palasantzas, G.; Krim, J. Phys. Rev. B 1993, 48, 2873.

16. Zhao, Y.-P.; Fortin, J. B.; Bonvallet, G.; Wang, G.-C.; Lu, T.-M. Phys. Rev. Lett. 2000, 85, 3229.

17. Collins, G. W.; Letts, S. A.; Fearon, E. M.; McEachern, R. L.; Bernat, T. P. Phys. Rev. Lett. 1994, 73, 708. 